\title{
APPLYING THE CZÉDLI-SCHMIDT SEQUENCES TO CONGRUENCE PROPERTIES OF PLANAR SEMIMODULAR LATTICES
}

\author{
G. GRÄTZER \\ Department of Mathematics \\ University of Manitoba \\ Winnipeg, MB R3T 2N2, Canada \\ e-mail: gratzer@me.com
}

\begin{abstract}
Following Grätzer and Knapp, 2009, a planar semimodular lattice $L$ is rectangular, if the left boundary chain has exactly one doubly-irreducible element, $c_{l}$, and the right boundary chain has exactly one doubly-irreducible element, $c_{r}$, and these elements are complementary.

The Czédli-Schmidt Sequences, introduced in 2012, construct rectangular lattices. We use them to prove some structure theorems. In particular, we prove that for a slim (no $\mathrm{M}_{3}$ sublattice) rectangular lattice $L$, the congruence lattice Con $L$ has exactly length $\left[c_{l}, 1\right]+\operatorname{length}\left[c_{r}, 1\right]$ dual atoms and a dual atom in Con $L$ is a congruence with exactly two classes. We also describe the prime ideals in a slim rectangular lattice.
\end{abstract}

Keywords: lattice, congruence, semimodular, planar, slim.

2010 Mathematics Subject Classification: Primary: 06C10, Secondary: 06B10.

\section{REFERENCES}

[1] K. Adaricheva, V.A. Gorbunov and V.I. Tumanov, Join-semidistributive lattices and convex geometries, Adv. Math. 173 (2003) 1-49. doi:10.1016/s0001-8708(02)00011-7

[2] S.P. Avann, Locally atomic upper locally distributive lattices, Math. Ann. 175 (1968) 320-336. doi:10.1007/bf02063217

[3] G. Birkhoff, Lattice Theory, Third Edition, American Mathematical Society Colloquium Publications, Vol. XXV (American Mathematical Society, Providence, R.I., 1967). 
[4] G. Czédli, A Horn sentence in coalition lattices, Acta Math. Hungarica 72 (1996) 99-104. doi: $10.1007 /$ bf00053700

[5] G. Czédli, Sums of lattices and a relational category, Order 26 (2009) 309-318. doi:10.1007/s11083-009-9127-7

[6] G. Czédli, A note on congruence lattices of slim semimodular lattices, Algebra Univ. 72 (2014) 225-230. doi:10.1007/s00012-014-0286-z

[7] G. Czédli, Finite convex geometries of circles, Discrete Math. 330 (2014) 61-75. doi:10.1016/j.disc.2014.04.017

[8] G. Czédli and G. Grätzer, Planar Semimodular Lattices: Structure and Diagrams, Chapter 3 in [25]. doi:10.1007/978-3-319-06413-0\_3

[9] G. Czédli, L. Ozsvárt and B. Udvari, How many ways can two composition series intersect?, Discrete Math. 312 (2012) 3523-3536.

doi:10.1016/j.disc.2012.08.003

[10] G. Czédli and E.T. Schmidt, Slim semimodular lattices I. A visual approach, Order 29 (2012) 481-497. doi:10.1007/s11083-011-9215-3

[11] R.P. Dilworth, Lattices with unique irreducible decompositions, Ann. of Math. 41 (1940) $771-777$. doi:10.1007/978-1-4899-3558-8\_10

[12] G. Grätzer, Lattice Theory: Foundation (Birkhäuser Verlag, Basel, 2011). doi:10.1007/978-3-0348-0018-1

[13] G. Grätzer, Planar Semimimodular Lattices: Congruences, Chapter 4 in [24]. doi:10.1007/978-3-319-06413-0\_4

[14] G. Grätzer, The Congruences of a Finite Lattice, A Proof-by-Picture Approach, Second Edition (Birkhäuser, 2016). doi:10.1007/978-3-319-38798-7

[15] G. Grätzer, Congruences in slim, planar, semimodular lattices: The Swing Lemma, Acta Sci. Math. (Szeged) 81 (2015) 381-397. doi:10.14232/actasm-015-757-1

[16] G. Grätzer, Congruences of fork extensions of lattices, Algebra Univ. 76 (2016) 139-154. doi:10.1007/s00012-016-0394-z

[17] G. Grätzer, Notes on planar semimodular lattices, VIII, Congruence lattices of SPS lattices, Algebra Univ. doi:10.1007/s00012-020-0641-1

[18] G. Grätzer and E. Knapp, Notes on planar semimodular lattices, I, Construction, Acta Sci. Math. (Szeged) 73 (2007) 445-462. Acta Sci. Math. (Szeged) 74 (2008) $37-47$. 
[19] G. Grätzer and E. Knapp, A note on planar semimodular lattices, Algebra Univ. 58 (2008) 497-499. doi:10.1007/s00012-008-2089-6

[20] G. Grätzer and E. Knapp, Notes on planar semimodular lattices, II, Congruences, Acta Sci. Math. (Szeged) 74 (2008) 37-47.

[21] G. Grätzer and E. Knapp, Notes on planar semimodular lattices, III, Rectangular lattices, Acta Sci. Math. (Szeged) 75 (2009) 29-48.

[22] G. Grätzer and E. Knapp, Notes on planar semimodular lattices, IV, The size of a minimal congruence lattice representation with rectangular lattices, Acta Sci. Math. (Szeged) 76 (2010) 3-26.

[23] G. Grätzer, H. Lakser, and E.T. Schmidt, Congruence lattices of small planar lattices, Proc. Amer. Math. Soc. 123 (1995) 2619-2623. doi: $10.2307 / 2160551$

[24] G. Grätzer and F. Wehrung eds., Lattice Theory: Special Topics and Applications, Volume 1 (Birkhäuser Verlag, Basel, 2014).

doi:10.1007/978-3-319-06413-0

[25] G. Grätzer and F. Wehrung eds., Lattice Theory: Special Topics and Applications, Volume 2 (Birkhäuser Verlag, Basel, 2016). doi:10.1007/978-3-319-44236-5

[26] D. Kelly and I. Rival, Planar lattices, Canad. J. Math. 27 (1975) 636-665. doi:10.4153/cjm-1975-074-0

[27] S. MacLane, A conjecture of Ore on chains in partially ordered sets, Bull. Amer. Math. Soc. 49 (1943) 567-568.

doi:10.1090/s0002-9904-1943-07972-4

[28] O. Ore, Chains in partially ordered sets, Bull. Amer. Math. Soc. 49 (1943) 558-566. doi:10.1090/s0002-9904-1943-07970-0

[29] P.M. Whitman, Free lattices, Ann. of Math. 42 (2) (1941) 325-330. doi:10.2307/1969001

[30] P.M. Whitman, Free lattices II, Ann. of Math. 43 (2) (1942) 104-115. doi: $10.2307 / 1968883$

Received 24 March 2020

Revised 4 December 2020

Accepted 6 December 2020 